\title{
Correlation between Serum Uric Acid Level and Left Ventricular Ejection Fraction in Patients with Heart Failure
}

\author{
Authors \\ Mohamed Yasar Arafath $\mathrm{S}^{\mathbf{1}}$, Senthilvelan $\mathbf{M}^{\mathbf{2}^{*}}$, Baburaj $\mathrm{K}^{\mathbf{3}}$ \\ ${ }^{1}$ Post Graduate, Department of General Medicine, Rajah Muthiah Medical College and Hospital, \\ Chidambaram, India, 608002 \\ ${ }^{2}$ Professor \& Head of the Department, Department of General Medicine, Rajah Muthiah Medical College \\ and Hospital, Chidambaram, India,608002 \\ ${ }^{3}$ Associate Professor, Department of General Medicine, Rajah Muthiah Medical College and Hospital, \\ Chidambaram, India, 608002 \\ *Corresponding Author \\ Dr Senthilvelan M
}

Professor \& Head of the Department, Department of General Medicine, Rajah Muthiah Medical College and Hospital, Chidambaram, India, 608002

\begin{abstract}
Background: The lifetime risk of heart failure is 20 - 45\% corresponding to age and race. Many studies in the past have debated whether uric acid as undependable risk factor in heart failure and its influence on left ventricular ejection fraction.

Objective: The aim of our study is to correlate the serum uric acid level and andit's influence on left ventricular ejection fraction in patients with established heart failure.

Methods: We conducted a prospective study that included 150 patients with established heart failure admitted in Rajah Muthiah Medical college and hospital based on selection criteria during the study period from October 2018 to October 2020.The study included patients with heart failure due to chronic arterial hypertension and coronary artery disease and it excludes patients with heart failure due to congenital heart disease, valvulopathies, severe anaemia, and patients with Gout, malignancy, and autoimmune diseases.

Results: There was a statistically significant $(p=0.001)$ correlation between serum uric acid level and Left Ventricular Ejection fraction but there is no significant correlation between uric acid and other risk factors such as Diabetes, systemic hypertension and smoking.

Conclusion: High levels of serum uric acid are significantly correlated with the low levels of ejection fraction and this study also indicate that uric acid is an important prognostic factor in heart failure.

Keywords: Uric Acid, Left Ventricular Ejection fraction, Heart failure.
\end{abstract}

\section{Introduction}

Uric acid is the end product of catabolism of purine nucleotides by xanthine oxidase. Xanthine oxidase and xanthine dehydrogenase catalyze the oxidation of hypoxanthine to xanthine which is the important enzyme in Purine metabolism and it leads to the generation of oxygen free radicals which increase the oxidative stress ${ }^{[1]}$. The role of 
uric acid as a cause of heart failure is still controversial. Oxidative stress along with nitric oxide disproportion may intensify the inflammatory pathways resulting in accelerated cytokine production ${ }^{[2]}$. Elevated Uric acid is related to many risk factors for heart failure like dyslipidemia and hypertension, indicating that uric acid might in turn be a marker of increased risk and this can induce disturbances of myocardial contractility, vasoconstriction and may negatively affect the cardiovascular system and significantly worsen the prognosis in patients with heart failure ${ }^{[3]}$. Further evidence of use of xanthine oxidase inhibitors to reduce serum uric acid levels showed improvement in cardiac function, endothelial activity and EF which lead to better results in heart failure. Several study shows that bioavailability of Nitric oxide derived from endothelium is increased by blocking the Xanthine Oxidase activity. In two randomised, placebo controlled double blind cross over study by George and colleagues ${ }^{[4]}$ proved that uricosuric agent probenecid showed no changes in endothelial function for same levels of decrease in uric acid in serum. ${ }^{[5]}$ There are certain studies about the correlation between serum uric acid and its influence on prognosis of heart failure and serum uric acid could be an important indicator of predicting prognosis in heart failure.

\section{Methods}

The study will be undertaken on the patients attending medicine inpatient department and admitted in Rajah Muthiah medical college and hospital, Chidambaram during the study period October 2018- October 2020. A total of 150 patients with heart failure are included in the study based on the inclusion and the exclusion criteria. Significant differences between serum uric acid in different subgroups to be observed over a period of 2 years and role of uric acid as a prognostic marker to be evaluated.

\section{Inclusion criteria}

- Heart failure due to chronic arterial hypertension
- Heart failure due to coronary artery disease

\section{Exclusion criteria}

Study excludes minors, pregnant women and following groups of patients with

- Congenital heart disease

- Valvulopathies

- Cardiomyopathies

- Chronic kidney disease

- Malignancy

- Gout

- Autoimmune disease

Ethics: The study is proposed to be conducted after obtaining informed consent from the patients. The duration of study is two year October 2018- October 2020. A detailed history, clinical examination and ECHO evaluation was done for all patients.

\section{Investigations}

1) Blood sample was collected from patients and analysed by standard methods for blood sugar, urea and creatinine.

2) Serum uric acid to be analysed by automatic chemical analyser.

\section{Results}

The study population of 150 patients were categorised based on age, Gender, NYHA classification and its influence on serum uric acid are statistically analysed.

Table 1: Age Distribution of the study populations

\begin{tabular}{|l|c|c|}
\hline $\begin{array}{l}\text { AGE } \\
\text { DISTRIBUTION }\end{array}$ & NUMBERS & PERCENTAGE \\
\hline $\mathbf{4 0 - 5 0}$ & 44 & 29 \\
\hline $\mathbf{5 1 - 6 0}$ & 55 & 37 \\
\hline $\mathbf{6 1 - 7 0}$ & 38 & 25 \\
\hline $\mathbf{7 1 - 8 0}$ & 10 & 7 \\
\hline$>\mathbf{8 0}$ & 3 & 2 \\
\hline
\end{tabular}


Age Distribution

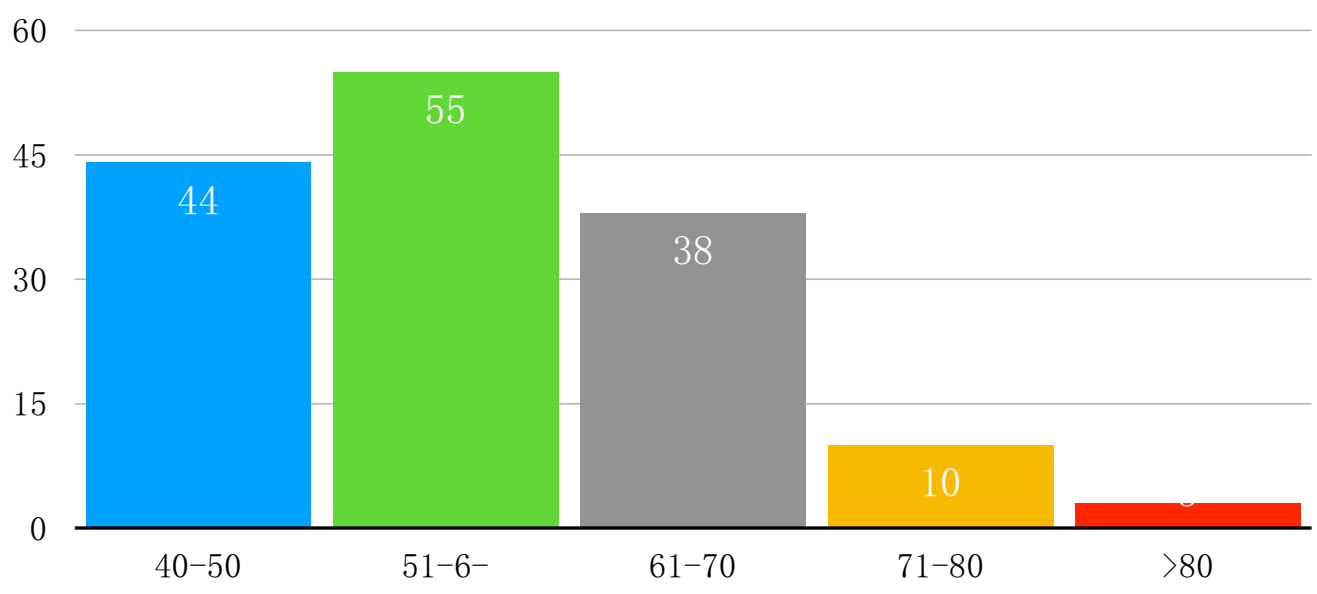

\section{Gender Distribution}

Study population contains $84 \%$ of males and $66 \%$ of females

Table 2: Gender Distribution

\begin{tabular}{|l|c|}
\hline SEX & NUMBER OF PATIENTS \\
\hline MALE & 84 \\
\hline FEMALE & 66 \\
\hline
\end{tabular}

$\because$ MALE $\quad$ FEMALE

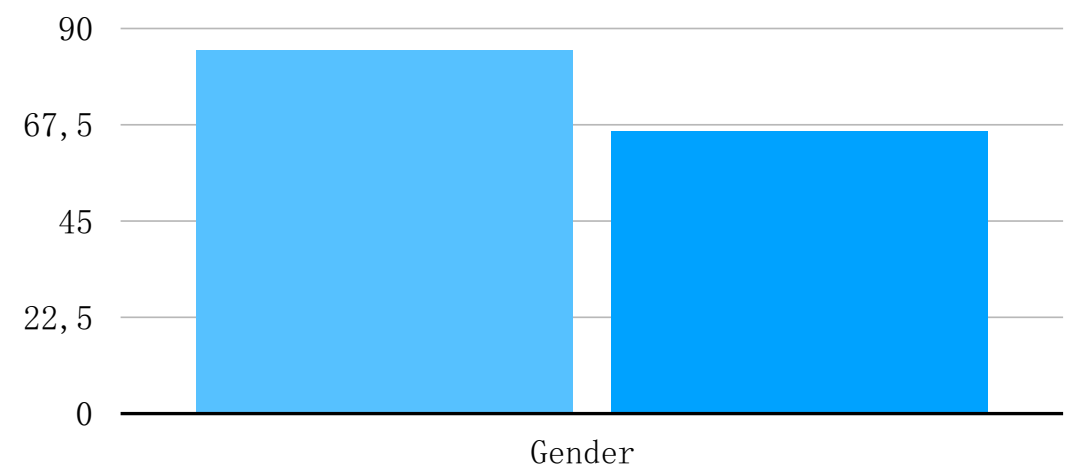

Distribution Based on NYHA Classification

Table 3: Distribution based on NYHA

\begin{tabular}{|l|c|c|}
\hline NYHA CLASS & NUMBER OF PATIENTS & PERCENTAGE \\
\hline I & 25 & 17 \\
\hline II & 63 & 42 \\
\hline III & 37 & 35 \\
\hline IV & 25 & 17 \\
\hline
\end{tabular}




\section{- NYHA}

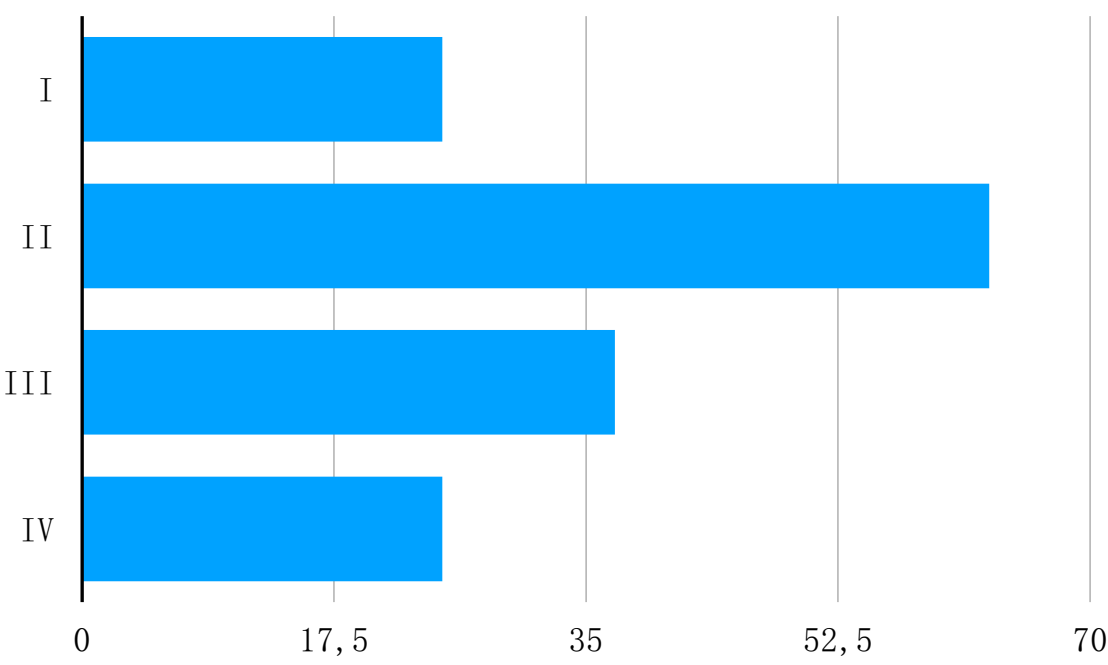

Distribution of Uric Acid

Table 4: Based on uric acid

\begin{tabular}{|l|c|}
\hline URIC ACID & NUMBER OF PATIENTS \\
\hline$>\mathbf{6 . 8}$ ( High) & 86 \\
\hline$<6.8$ (Normal) & 64 \\
\hline
\end{tabular}

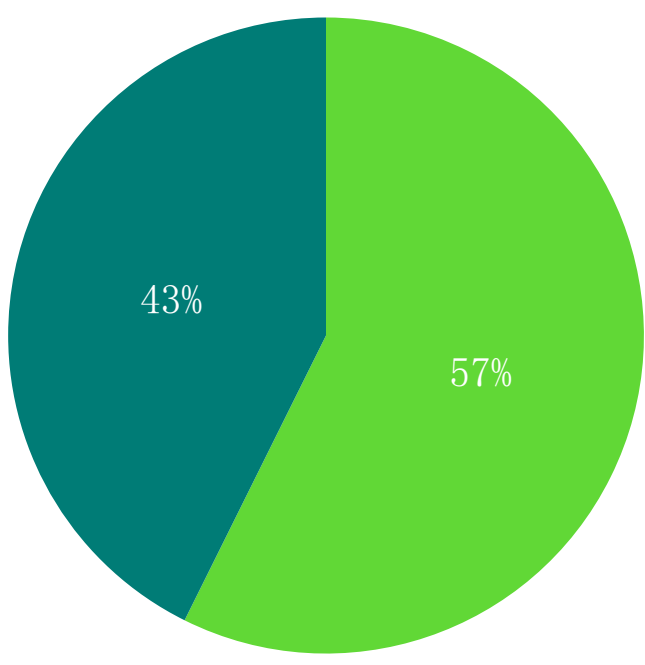

Correlation between Serum Uric Acid and Ejection Fraction

Table 5: Correlation between EF and serum uric acid

\begin{tabular}{|l|c|c|c|c|c|c|}
\hline \multirow{2}{*}{$\begin{array}{l}\text { URIC ACID } \\
(\mathbf{m g} / \mathbf{d l})\end{array}$} & \multicolumn{5}{|c|}{ Ejection fraction } & \multicolumn{2}{c|}{ Overall } \\
\cline { 2 - 7 } & \multicolumn{2}{|c|}{$<\mathbf{4 0}$} & \multicolumn{4}{c|}{} \\
\hline & Numbers & Percentage & Numbers & Percentage & Numbers & Percentage \\
\hline$<6.8$ & 18 & 29 & 68 & 77.3 & 86 & 57.3 \\
\hline$>\mathbf{6 . 8}$ & 44 & 71 & 20 & 22.7 & 64 & 42.7 \\
\hline
\end{tabular}




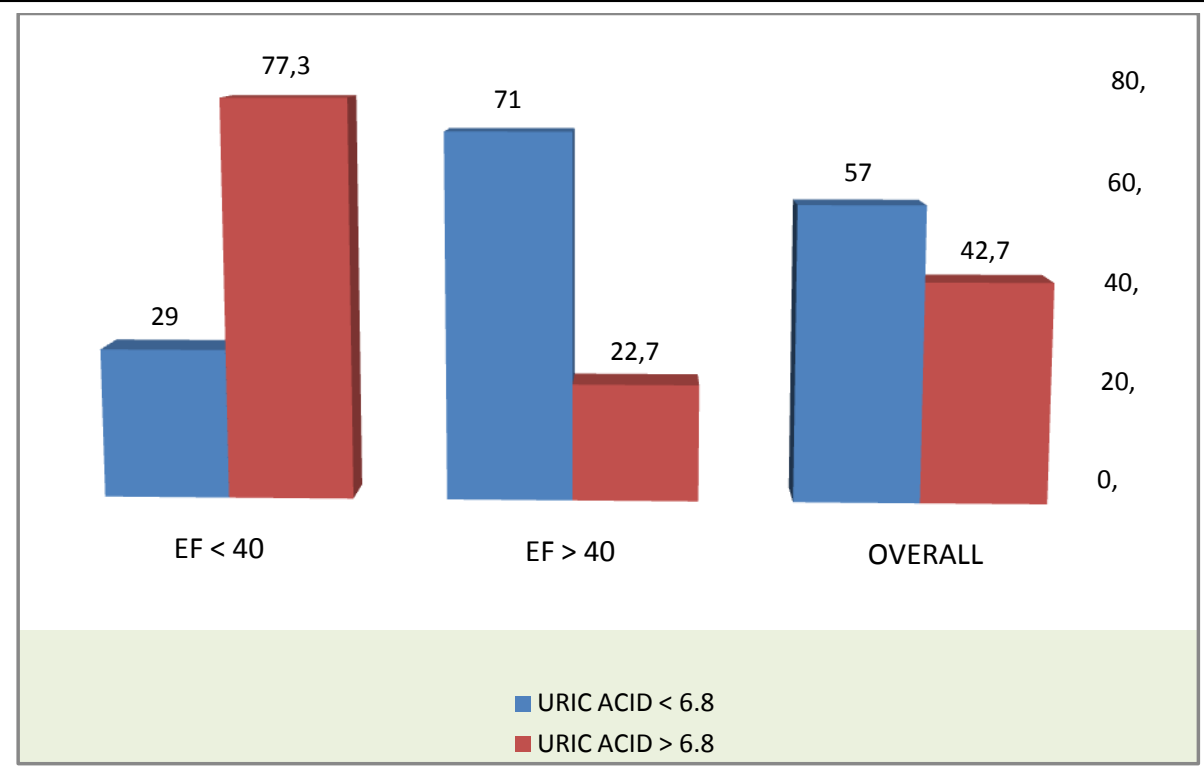

It is observed that, in patients with ejection fraction $<40,71 \%$ of them had high uric acid(> $6.8 \mathrm{mg} / \mathrm{dl}$ ) whereas in patients with ejection fraction $>40$ only $22.7 \%$ had high uric acid levels. The difference was statistically significant, $\mathrm{x}^{2}=34.6, \mathrm{P}=0.001$.

\section{Correlation between Uric Acid and Prognosis}

\begin{tabular}{|c|c|c|c|c|c|c|c|c|}
\hline \multirow{3}{*}{ Prognosis } & \multicolumn{4}{|c|}{ Serum Uric Acid } & \multirow{2}{*}{\multicolumn{2}{|c|}{ Overall }} & \multirow{2}{*}{\multicolumn{2}{|c|}{ CHI-SQUARE }} \\
\hline & \multicolumn{2}{|c|}{$<6.8$} & \multicolumn{2}{|c|}{$>6.8$} & & & & \\
\hline & Numbers & Percentage & Numbers & Percentage & Numbers & Percentage & Value & $P$ value \\
\hline Dead & 4 & 6.25 & 22 & 25 & 26 & 17.3 & & \\
\hline Alive & 60 & 93.75 & 64 & 75 & 124 & 82.7 & & \\
\hline
\end{tabular}

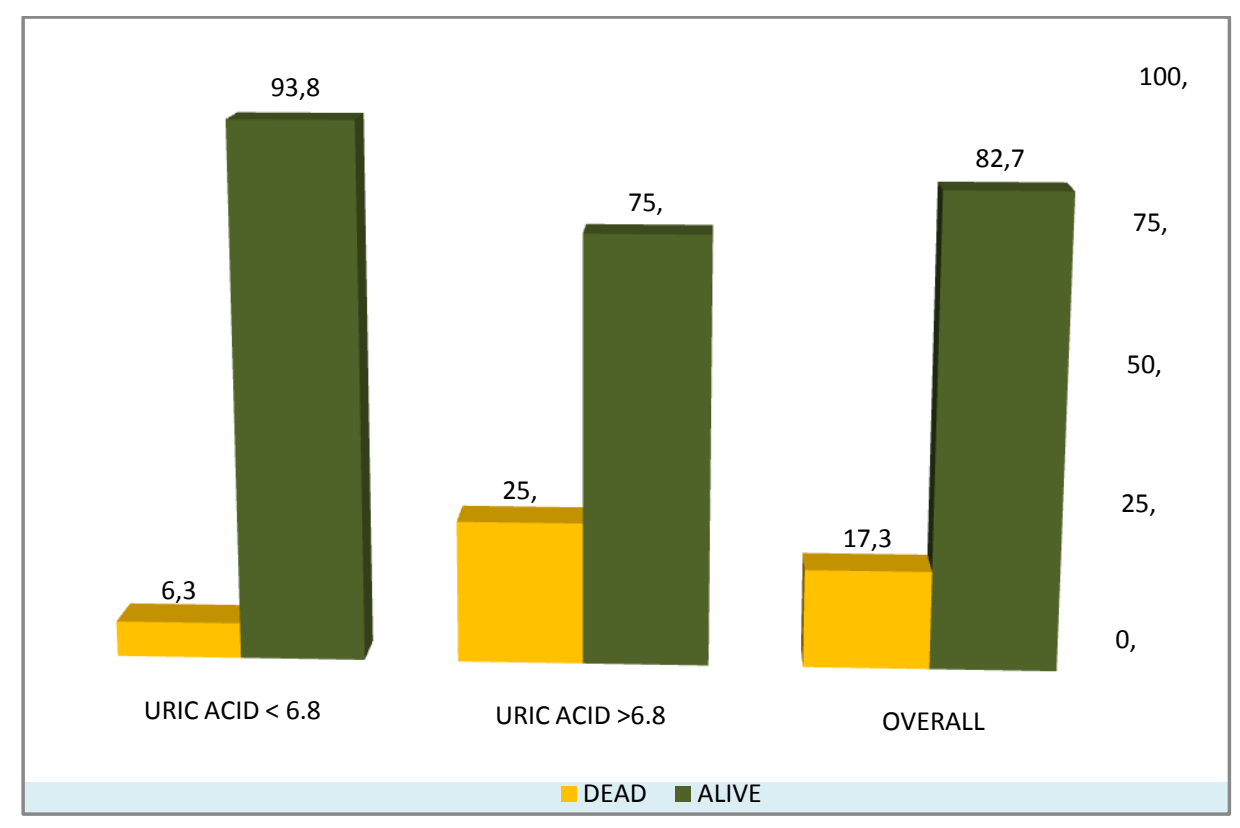

It is observed that high uric acid is associated with poor prognosis in patients with heart failure and it is statistically significant, $p=0.001$ 


\section{Discussion}

Uric acid as a risk factor heart failure is still under debate whether it's an in dependable risk factor or it may be as a result of other disorders like diabetes and hypertension ${ }^{[6]}$. The probable effect of high uric acid level in the failing heart is due to its association with dysfunction of endothelium, increased oxidative stress and formation of thrombus ${ }^{[8]}$. Data from Beta Blocker Evaluation of Survival Trial $^{[7]}$ took a different approach assuming that hyperuricemia without chronic renal failure is primarily due to increased production of UA from the failing heart. The conclusion in that study was hyperuricemia was associated with poor outcomes in heart failure without renal failure. In our study most of them are hyperuricemia with percentage of $57 \%$ while $43 \%$ are non-hyperuricemic .In our study, the group of patients with high uric acid (>6.8) around $71 \%$ of them have low ejection fraction $(<40)$.Our study revealed that there is significant association between serum uric acid and left ventricular ejection fraction which was in agreement with the study by Deveci et al ${ }^{[9]}$.

\section{Conclusion}

Our study concluded that serum uric acid is negatively correlation with Left ventricular Ejection fraction with $\mathrm{p}$ value $<0.001$.Serum uric acid may be considered as an independent risk factor for heart failure and also as a prognostic marker in heart failure.

Conflicts of Interest: The authors declare no conflicts of interest regarding the publication of this paper.

\section{References}

1. McMurray, J.J.V., Cherubini, A., Ble, A., Bos, A.J.G., Maggio, M., Dixit, V.D., et al. (2014) Uric Acid and Inflammatory Markers. European Heart Journal, 27, 11741181.

https://doi.org/10.1093/eurheartj/ehi879.
2. Anker, S.D., Granner, D.K., Mayes, P.A. and Rodwell, V.W. (2012) Harper's Illustrated Biochemistry. Molecular Physiology.

3. Adamopoulos, S., Anker, S.D., Auricchio, A., Böhm, M., Dickstein, K., et al. (2012) ESC Guidelines for the Diagnosis and Treatment of Acute and Chronic Heart Failure. European Heart Journal, 33, 1787-1847.

4. Ogino K, Kato M, Furuse Y, et al. UAlowering treatment with benzbromarone in patients with HF a double-blind placebocontrolled crossover preliminary study. Circulation: HF. 2010;3:73-81.

5. Leyva, F., Anker, S.D., Godsland, I.F., Teixeira, M., Hellewell, P.G., Kox, W.J., et al. (1998) Uric acid in Chronic Heart Failure: A Marker of Chronic Inflammation. Eu- ropean Heart Journal, 19, 1814-1822. https://doi.org/10.1053/euhj.1998.1188.

6. Harzand, A., Palacio, A., Verma, S., Jones, J., Hare, J., et al. (2016) Uric Acid as a Predictor of All-Cause Mortality in Heart Failure: A Meta-Analysis. Congestive Heart Failure, 17, 25-30. https://doi.org/10.1111/j.17517133.2011.00200.x.

7. Deveci, T., Takeishi, Y., Arimoto, T., Okuyama, H., Nozaki, N., Hirono, O., et al. (2013) Hyperuricemia Associated with High Cardiac Event Rates in the Elderly with Chronic Heart Failure. Journal of Cardiology, 47, 219-228.

8. Filippatos GS, Ahmed MI, Gladden JD, Aban IB, Love TE, Sanders PW, et al. Hyperuricemia, chronic kidney disease, and outcomes in heart failure: potential mechanistic insights from epidemiological data. Eur Hear J. 2011;32(6):712-20.

9. Deveci, O.S., Kabakci, G., Okutucu, S., Tulumen, E., et al. (2010). The Association between Serum Uric Acid Level and Coronary Artery Disease. ClinPract, 64, 900-907. 